\title{
Outbreak of laboratory-acquired Brucella abortus in Brazil: a case report
}

\author{
Ana Luisa Calixto Rodrigues ${ }^{[1]}$, Stéphanie Kneipp Lopes da Silva ${ }^{[1]}$, \\ Bárbara Luíza Alves Pinto[ ${ }^{[1]}$, Jane Braga da Silva ${ }^{[1]}$ and Unaí Tupinambás ${ }^{[2]}$
}

[1]. Curso de Medicina, Universidade Federal de Minas Gerais, Belo Horizonte, MG. [2]. Departamento de Clínica Médica, Universidade Federal de Minas Gerais, Belo Horizonte, MG.

\begin{abstract}
Human brucellosis is an occupational disease affecting workers in slaughterhouses, butcher shops and the milk and dairy product industry as well as individuals who work in clinical or research laboratories. We report the first outbreak of a Brucella abortus infection in a Brazilian laboratory and compare the data obtained with reports available in the literature. Exposure was a result of damage to a biological safety cabinet and failure of the unidirectional airflow ventilation system. An epidemiological investigation identified 3 seroconverted individuals, 1 of whom had clinical manifestations and laboratory results compatible with infection at the time of exposure $(\mathrm{n}=11$; attack rate $=9.1 \%)$.
\end{abstract}

Keywords: Laboratory-acquired infection. Brucella abortus. Occupational exposure. Aerosolization.

\section{INTRODUCTION}

Human brucellosis is an occupational disease affecting individuals exposed to Brucella spp. in contexts in which animal infection occurs ${ }^{1,2}$, and numerous cases of brucellosis acquired in clinical or research laboratories have been reported ${ }^{3,4}$. However, no published accounts of laboratory-acquired brucellosis in Brazil were found in PubMed/Medline and Scielo searches up to the date of submission. This paper provides the first report of an outbreak of Brucella abortus infection in a Brazilian laboratory, and it compares the data obtained with the reports available in the literature. Data were obtained through the administration of questionnaires to all of the exposed individuals and from their laboratory test results $(\mathrm{n}=11)$.

Brucella is a genus of Gram-negative coccobacilli. Human brucellosis is caused by B. melitensis, B. abortus, $B$. suis and $B$. canis. The most common symptoms are fever, sweating, headache, myalgia, vomiting, weakness and loss of weight. Frequent clinical findings include hepatomegaly and splenomegaly. Osteoarticular manifestations can occur, including sacroiliitis, spondylitis and osteomyelitis. Infection with Brucella spp. does not always result in clinical manifestations ${ }^{1}$.

In Brazil, brucellosis mainly affects cattle, and the most common agent is B. abortus ${ }^{5}$. Human brucellosis cases occur through transmission from contaminated food or in the work

Address to: Dr. Unaí Tupinambás. Depto Clínica Médica/UFMG. Av. Alfredo Balena 190, 30130-100 Belo Horizonte, MG, Brasil.

Phone: 5531 3409-9822/55 31 9979-3052; Fax: 5531 3273-0422

e-mail: unaitupi@gmail.com

Received 18 August 2012

Accepted 21 November 2013 environment. Food-borne transmission occurs due to the ingestion of unpasteurized milk and other contaminated dairy products $^{2}$, but because of the compulsory pasteurization of milk products and strict control of the disease in dairy cattle, foodborne human brucellosis is rare in Brazil ${ }^{1}$.

Occupational brucellosis can be divided into 2 categories. The first category comprises infections acquired in slaughterhouses and butcher shops, during the handling of meat products, and in the milk and dairy product industry. Veterinary physicians, animal caretakers, butchers and slaughterhouse workers form the corresponding risk group ${ }^{1,2}$. Transmission of the bacteria to human beings occurs through cuts on the skin and direct contact with tissues from contaminated animals ${ }^{2}$. The second category of occupational brucellosis includes those infections acquired in clinical and research laboratories, with microbiologists and laboratory staff members in the corresponding risk group ${ }^{1,3,6}$. Laboratory-acquired infections represent $2 \%$ of all brucellosis cases worldwide ${ }^{3}$.

Brucella spp. are some of the most common pathogens responsible for laboratory-acquired infections, along with Shigella, Salmonella, Mycobacterium tuberculosis and Neisseria meningitides ${ }^{7,8}$. Of these pathogens, laboratory-acquired Brucella poses the greatest relative risk: a study found that the incidence of Brucella infection was 641 cases per 100,000 laboratory technologists, compared with 0.08 cases per 100,000 persons in the general population ${ }^{8}$. The attack rate after laboratory exposure ranges from $30 \%$ to $100 \%{ }^{3}$. Most Brucella laboratory infections are caused by $B$. melitensis ${ }^{6,9}$.

In laboratories, exposure occurs due to aerosolization, followed by inhalation, during routine identification activities in $88 \%$ of cases, according to a recently published review ${ }^{4}$, but the bacterium can also be transmitted via direct contact $^{8,9,10}$. Laboratory accidents, such as the breakage of centrifuge tubes or blood-culture vials, are not frequent and represent only $11 \%$ 
of cases ${ }^{4}$. In most of the reported cases, exposure resulted from unsafe laboratory practices, such as working on an open bench with viable organisms; sniffing plates; not using protective equipment, such as gloves, masks and goggles; or ingesting suspensions of living Brucellae during mouth pipetting ${ }^{3}$.

The concentration of Brucella organisms in the blood and synovial fluid of patients with brucellosis is usually low. Therefore, clinical specimens most likely pose a low risk of contagion for laboratory personnel. However, the danger of clinically relevant exposure increases significantly after incubation of seeded media ${ }^{3}$. Bacteriological procedures that generate aerosols, procedures with live bacteria, being male, handling of seeded media outside biological safety cabinets and poor compliance with hand hygiene and with the wearing of personal protection equipment have previously been identified as independent risk factors for laboratory-acquired brucellosis ${ }^{9,10}$.

Thus, work with Brucella cultures is restricted to biosafety level 3 (BSL 3) laboratories ${ }^{1}$. This type of facility requires the handling of microbial cultures inside laminar flow biological safety cabinets (BSCs), i.e., enclosed, ventilated laboratory devices for working with pathogens. Access to the BSL 3 facility is gained through 2 self-closing doors, with an airlock clothes-changing room (or anteroom) between them. A ducted air ventilation system is required to provide sustained unidirectional airflow by drawing air into the laboratory from non-contaminated areas toward potentially contaminated areas, so the laboratory exhaust air does not re-circulate. For this purpose, vacuum lines must be installed and protected with high-efficiency particulate absorption (HEPA) air filters ${ }^{11}$.

\section{CASE REPORT}

In late May 2012, heavy rain damaged a BSC in a BSL 3 laboratory in which media seeded with B. abortus had been manipulated. The opening in the BSC was subsequently covered with canvas to protect the device from rainwater. After the incident, laboratory staff realized that the unidirectional ventilation system was not functioning. The risk of infection was immediately identified, and an epidemiological investigation was initiated.

The population exposed to B. abortus comprised all of the persons who entered the laboratory and/or its anteroom during the period between the accident and the identification of the ventilation system failure. In total, 11 individuals were included in the investigation: 10 (90.9\%) were female members of laboratory staff, and the individuals were between 23 and 37 years old in May 2012. All of them reported wearing laboratory coats, disposable gloves and N95 masks regularly when they were inside the BSL 3 facility, and all of them denied any previous history of Brucella infection.

All exposed individuals were clinically monitored and subjected to serological testing (enzyme-linked immunosorbent assay - ELISA). Seven (63.6\%) individuals reported having repeated the test after a period of 1 or 2 months for confirmation of the results. Three laboratory workers had reactive $\operatorname{IgM} / \operatorname{IgG}$ antibodies, identified either by the 1st (workers 1 and 2) or the subsequent (worker 3) ELISA tests performed after the incident (Table 1).

TABLE 1 - Results from serological (ELISA) and molecular testing PCR among the individuals exposed to Brucella abortus.

\begin{tabular}{lcc}
\hline & \multicolumn{2}{c}{ Number of patients } \\
\cline { 2 - 3 } Test result & $1^{\text {st }}$ exam (n) & subsequent exams (n) \\
\hline ELISA IgG/IgM reactive & $2(11)$ & $1(5)$ \\
PCR-positive & $0(3)$ & - \\
\hline
\end{tabular}

ELISA: enzyme-linked immunosorbent assay; PCR: polymerase chain reaction; IgG/IgM: immunoglobulin G/immunoglobulin M. The subsequent exam results only included patients who had negative results based on the first exam.

\section{Worker 1}

A 34-year-old female laboratory worker reported general unwellness and cephalalgia after her exposure. Two consecutive ELISA tests, performed on July 8 and August 23, 2012, were negative for immunoglobulin $\mathrm{M}$ (IgM) but indicated reactive immunoglobulin $\mathrm{G}(\operatorname{IgG})$ antibodies against Brucella. Despite the absence of other symptoms, prophylactic treatment with doxycycline and rifampicin was initiated on July 18 and continued for 42 days.

\section{Worker 2}

A 28-year-old male laboratory worker presented symptoms shortly after exposure. He reported fever, general unwellness, chills, cephalalgia, excessive sweating and arthralgia (lower back pain). His clinical examination indicated osteoarticular involvement, and a diagnostic hypothesis of sacroiliitis was confirmed by magnetic resonance imaging (MRI) (Figure 1). On June 30, 2012, he had IgM-positive ELISA results, his C-reactive protein level was $45.4 \mathrm{mg} / \mathrm{L}$, and his erythrocyte sedimentation rate was $65 \mathrm{~mm}$ in 60 minutes. On July 6, the patient was hospitalized, and treatment with doxycycline and rifampicin was initiated. Hospitalization and antibiotic treatment lasted 10 and 56 days, respectively. As of July 31, his ELISA results were still IgM-positive, but on August 23, the ELISA index was undetermined. Polymerase chain reaction (PCR) results were negative on October 10. The patient responded well to the treatment, and ELISA results were IgM/IgG-negative in February 2013.

\section{Worker 3}

A 29-year-old female laboratory worker reported general unwellness after the accident in the NB3 facility. Her first ELISA result, on July 10, 2012, was both IgM- and IgG-negative, but a subsequent test on October 24 that was negative for $\operatorname{IgG}$ antibodies showed an IgM-undetermined index. The patient presented no other relevant symptoms, and no prophylactic treatment was established. Then, on February 7, 2013, a new 


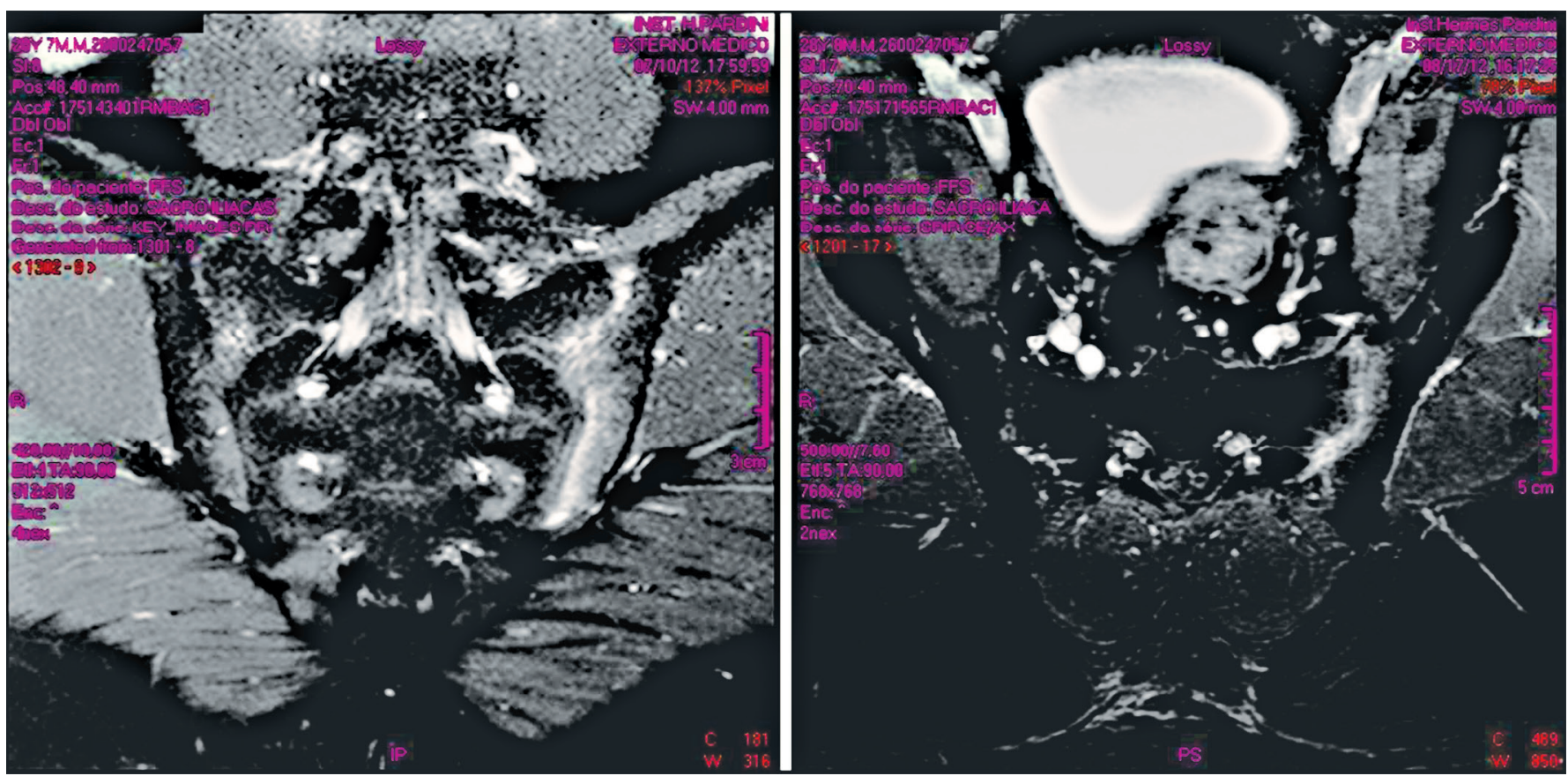

FIGURE 1 - Sacroiliitis diagnosis based on MRI. T2-weighted MRI with fat saturation, showing a hypersignal on the left sacroiliac joint and bone edema on both the coronal and the axial planes. MRI: magnetic resonance imaging.

ELISA test was IgM-negative/IgG-positive. Despite the absence of clinical manifestations, chemoprophylaxis with gentamicin + doxycycline and rifampicin was established. This patient is currently undergoing periodical clinical monitoring.

\section{DIscussion}

Only worker 2 had clinical, epidemiological and laboratory data that clearly supported a confirmed Brucella infection acquired by exposure in May 2012. For workers 1 and 3, the association of seroconversion with the same exposure event was supported by the incubation period after exposure to Brucella varying considerably, ranging from 5 to 60 days in most cases but possibly extending to several months ${ }^{1}$, and both individuals denying a history of past Brucella infection. However, the clinical manifestations for workers 1 and 3 were unspecific, and the ELISA tests could not exclude previous infection for worker 1 or re-exposure of worker 3 . Therefore, only worker 2 was considered a confirmed case. Accordingly, the attack rate associated with exposure after the event in May 2012 was $1 / 11(9.1 \%)$.

In the case of worker 2 , infection could be associated with the incident that generated a failure of the unidirectional ventilation system. Transmission most likely occurred from an aerosol-generating event, as is commonly reported. However, contrary to most published accounts ${ }^{3,4}$, transmission was related to an accident, and no correlation with unsafe routine procedures could be established. No other reports of Brucella transmission associated with ventilation system failures were found.
The Brazilian Ministry of Health ordinance regarding workrelated diseases (Portaria 1339/1999) describes brucellosis as a zoonosis caused by occupational exposure due to activities at slaughterhouses and butcher shops, the handling of meat products, the milk and dairy product industry and similar activities $^{12}$. There is no mention of laboratory exposure. Nonetheless, as demonstrated by this case report, transmission in laboratories has already occurred in Brazil. Thus, public policies concerning occupational diseases should extend to laboratory-acquired brucellosis.

This first report of laboratory-acquired human brucellosis in Brazil demonstrates that laboratory transmission is part of the occupational dimension of brucellosis. Furthermore, it emphasizes the need to continue epidemiological supervision and the importance of adequate biological safety measures for the handling of Brucella organisms, particularly the functioning of unidirectional airflow mechanisms.

\section{REFERENCES}

1. Laxinsky MLJ, Ohara PM, Elkhoury MR, Faria NC, Cavalcante KRLJ Estado da arte da brucelose em humanos. Rev Pan-Amaz Saude 2010; 4:75-84.

2. Robinson A. Guidelines for coordinated human and animal brucellosis surveillance (FAO animal production and health paper 156). Rome: Food and Agriculture Organization; 2003. 45p.

3. Yagupsky P, Baron EJ. Laboratory Exposures to Brucellae and Implications for Bioterrorism. Emerg Infect Dis 2005; 11:1180-1185.

4. Traxler RM, Lehman MW, Bosserman EA, Guerra MA, Smith TL. A literature review of laboratory-acquired brucellosis. J Clin Microbiol $2013 ; 51: 3055-3062$. 
5. Poester FP, Gonçalves VSP, Lage AP. Brucellosis in Brazil. Vet Microbiol 2002; 90:55-62.

6. Gruner E, Bernasconi E, Galeazzi RL, Buhl D, Heinzle R, Nadal D. Brucellosis: an occupational hazard for medical laboratory personnel. Infection 1994; 22:33-36.

7. Baron EJ, Miller JM. Bacterial and fungal infections among diagnostic laboratory workers: evaluating the risks. Diag Microbiol Infect Dis 2008; 60:241-246.

8. Singh K. Laboratory-Acquired Infections. Clin Infect Dis 2009; 49: 142-147.

9. Ergönül Ö, Çelikbas A, Tezerenb D, Güvener E, Dokuzoguz B. Analysis of risk factors for laboratory-acquired brucella infections. J Hosp Infect $2004 ; 56: 223-227$
10. Sayin-Kutlu S, Kutlu M, Ergönül Ö, Akalin S, Guven T, Demiroglu YZ, et al. Laboratory-acquired brucellosis in Turkey. J Hosp Infect 2012; 80:326-330.

11. Centers for Disease Control and Prevention. Biosafety in Microbiological and Biomedical Laboratories. $5^{\text {th }}$ ed. Atlanta: Centers for Disease Control and Prevention, 2009. 438 p.

12. República Federativa do Brasil. Ministério da Saúde. Portaria No. 1339/ GM em 18 de novembro de 1999. Anexo: lista de doenças relacionadas com o trabalho do Ministério da Saúde. 